\title{
Real Time HCI and Limits of Human Performance
}

\author{
V. Risak ${ }^{1}$
}

Summary: Real-time-systems impose demands concerning complexity and reaction-fime onto the user. The limits of human performance can be compared with these demands in a special diagram. Special types of handicaps and the result of countermeasures by modifying the task or training can be shown.

\section{Introduction:}

The difficulty of real-time tasks is essentially influenced by complexity and necessary reaction-time. Both components alone or in combination have to be compared with the limits of human performance. Examples are: driving a racing-car (reaction-time), playing chess (complexity) or controlling an industrial plant (complexity and reaction time).

Handicaps can cause longer reaction-times and-or reduce the information-processing ability. Countermeasures try to compensate these handicaps by lowering the real-time demands (e.g. by automating some functions like ABS in cars), by reducing the complexity (e.g. automating routine tasks like automatic switch-gear, intelligent-help with operators-guide) or by special training (e.g. with simulators) to relief the scarce resource of conscious information-processing.

\section{Complexity-Velocity-Diagram}

We propose a diagram, which allows us to compare the human performance-limits with the difficulty of the task. The effect of different types of handicaps relative to a given task can be shown. If this task is too difficult, possible countermeasures can be derived.

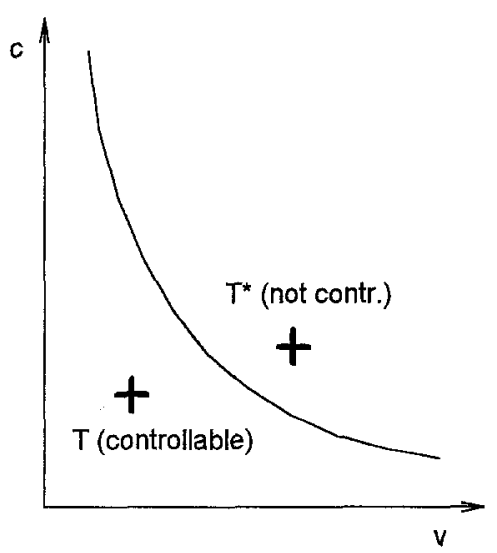

Fig. 1 c-v-Diagram We will assume, that the performance-limit $P$ of a given person depends on his velocity $v$ and his fitness to handle complexity $c$.

$$
\begin{aligned}
& P(v, c)=k \cdot\left(v^{a} \cdot c^{b}\right) \\
& a, b, k>0
\end{aligned}
$$

We use this product-form, because $P=0$ either if the velocity of reaction $v=0$ or the ability to manage complexity $c=0$.

The exponents $a$ and $b$ make it possible to give different weights to both components of human performance.

\footnotetext{
${ }^{1}$ Siemens Austria PSE1, University of Salzburg, risak@siemens.co.at
} 
If $a=b$ both components have equal weight. If $a>b(a<b)$ than the reaction velocity is better (worse) than the management of complexity. $k$ is a measure of overall performance, whilst $a$ and $b$ enhance selectively one or the other performance component.

(1) takes the form of hyperbolas ${ }^{2}$, which by $k, a, b$ describe the abilities of a person. ${ }^{3}$ The area below the hyperbola is controllable by him (e.g. a task marked by T). The task $\mathrm{T}^{*}$ above the hyperbola is too hard.

\section{Types of Handicaps}

Handicaps reduce the performance in some types of tasks. In the diagram the corresponding hyperbola lies below the original one. We distinguish:

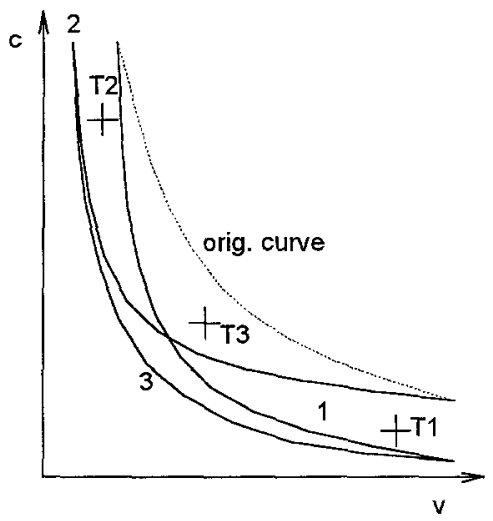

Fig. 2. Types of handicaps

- Reaction-velocity handicaps: (Fig. 2, curve 1) The velocity-parameter $a$ is lowered. As a consequence performance on time-critical tasks is impaired, whilst the ability to manage complexity is normal. (This corresponds to peripheral handicaps with intact intelligence.)

- Complexity Handicaps: (Fig. 2, curve 2)

The complexity-parameter $b$ is lowered. The management of complexity is reduced, but reaction-velocity is normal. That means, easy tasks can be handled as before, but growing complexity early cause overload. (This corresponds to reduced intelligence with normal peripheral reaction-velocity.)

- Global Symmetrical Handicaps: (Fig. 2, curve 3)

The global performance-parameter $k$ is reduced. The corresponding hyperbola lies nearer to the origin. Both reaction-velocity and handling of complexity are reduced. $^{4}$

The consequences for mastering tasks can be seen from the relative position of the corresponding points describing their difficulty.

\footnotetext{
2 It could be better to use a log-scale on both axes, because the hyperbolas then look like straight lines. But there is the problem, that the complexity of tasks varies for many orders of magnitude, whilst reaction-velocity does not vary so much.

${ }^{3}$ For more realistic purposes we could define an absolute upper limit on reaction velocity caused by anatomical conditions, e.g. reaction-time $>0,1 \mathrm{sec}$. For complexity, given enough time, we see no such limit.

4 I think this type of handicap is not very realistic, because velocity- and complexityhandicaps follow different mechanisms.
} 


\section{Counter-Measures:}

After describing different types of handicaps, we will discuss compensating measures.

One possibility is to make the task easier, so that the task-point lies below the performance hyperbola. This can be accomplished by (see Fig. 3):

- reducing the complexity (arrow 1) for example by automating routine-tasks, intelligent help, expert-systems, working on a higher abstraction level, ...

- reducing reaction-velocity demands (arrow 2 ) for example by automating time-critical sub-tasks like ABS, ...

Another way is to enhance the capabilities of the user:

This can be done e.g. by systematic training. After sufficient training we can perform actions unconscious, which before had to done conscious step by step. This relieves the load on the very scarce resource conscious-thinking. This free capacity now can be used otherwise, c.g. for managing complexity.

An example is learning to drive a car in heavy traffic:

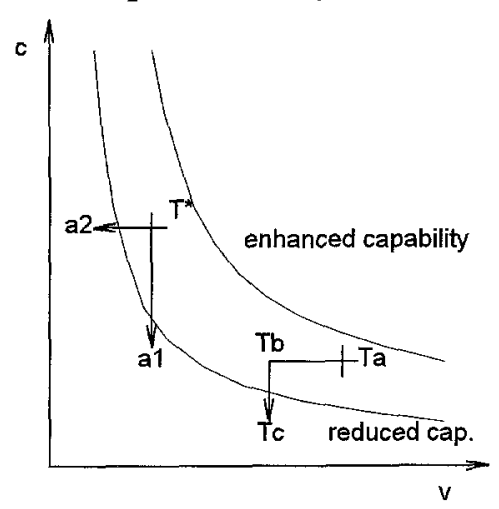

In the beginning this task (point $\mathrm{Ta}$ ) is without reach of the learner. Therefore the first steps are performed slowly (reduced reaction-velocity needed, point $\mathrm{Tb}$ ) on an empty place (reduced complexity) The task-point is now moved below the hyperbola (point Tc). After some experience the individual global performance-parameter $k$ grows, so that in the end the demands of real heavy traffic (point $\mathrm{Ta}$ ) now can be mastered.

Fig. 3. Counter-measures

\section{Summary:}

A qualitative complexity/velocity-diagram gave us the possibility to display and compare limits of human performance at the man-machine-interface. We described different types of handicaps and discussed the effect of possible counter-measures. Perhaps this diagram gives us the possibility to locate main problems in real-time situations and to help in selecting the proper measures. 